\title{
Efficacy of Non-Beta-lactam Antibiotics for Prevention of Cesarean Delivery Surgical Site Infections
}

\author{
Benjamin S. Harris, MD, MPH ${ }^{1}$ Maeve K. Hopkins, MD ${ }^{1}$ Margaret S. Villers, MD, MSCR ${ }^{2}$ \\ Jeremy M. Weber, MS ${ }^{3}$ Carl Pieper, DrPH ${ }^{3}$ Chad A. Grotegut, MD, MHSc, MBA ${ }^{2}$ Geeta K. Swamy, MD ${ }^{2}$ \\ Brenna L. Hughes, MD, $\mathrm{MSC}^{2}$ R Phillips Heine, $\mathrm{MD}^{2}$
}

${ }^{1}$ Department of Obstetrics and Gynecology, Duke University Health System, Durham, North Carolina

2 Division of Maternal Fetal Medicine, Department of Obstetrics and Gynecology, Duke University Health System, Durham, North Carolina

${ }^{3}$ Department of Biostatistics and Bioinformatics, Duke University Health System, Durham, North Carolina
Address for correspondence Benjamin Harris, MD, MPH, Department of Obstetrics and Gynecology, Duke University Health System, Durham, NC 27710 (e-mail: benjamin.harris@duke.edu).

Am J Perinatol Rep 2019;9:e167-e171.

\begin{abstract}
Keywords

- antibiotics

- penicillin allergy

- cesarean delivery

- surgical site infection

Objective To examine the association between perioperative Beta ( $\beta$ ))-lactam versus non- $\beta$-lactam antibiotics and cesarean delivery surgical site infection (SSI).

Study Design Retrospective cohort of women undergoing cesarean delivery from January 1 to December 31, 2014. All women undergoing cesarean after 34 weeks with a postpartum visit were included. Prevalence of SSI was compared between women receiving $\beta$-lactam versus non- $\beta$-lactam antibiotics. Bivariate analyses were performed using Pearson's Chi-square, Fisher's exact, or Wilcoxon's rank-sum tests. Logistic regression models were fit controlling for possible confounders.

Results Of the 929 women included, $826(89 \%)$ received $\beta$-lactam prophylaxis and $103(11 \%)$ received a non- $\beta$-lactam. Among the 893 women who reported a non-type I (low risk) allergy, $819(92 \%)$ received $\beta$-lactam prophylaxis. SSI occurred in $7 \%$ of women who received $\beta$-lactam antibiotics versus $15 \%$ of women who received a non- $\beta$-lactam $(p=0.004) . \beta$-Lactam prophylaxis was associated with lower odds of SSI compared with non- $\beta$-lactam antibiotics (odds ratio $[\mathrm{OR}]=0.43 ; 95 \%$ confidence interval $[\mathrm{Cl}]$ $=0.22-0.83 ; p=0.01$ ) after controlling for chorioamnionitis in labor, postlabor cesarean, endometritis, tobacco use, and body mass index (BMI).

Conclusion $\beta$-Lactam perioperative prophylaxis is associated with lower odds of a cesarean delivery surgical site infection compared with non- $\beta$-lactam antibiotics.
\end{abstract}

Over one-third of women in the United States delivered via cesarean delivery (CD) in 2015 and approximately 2 to $18 \%$ of CDs are complicated by surgical site infections (SSI). ${ }^{1-5} \mathrm{SSI}$ leads to significant morbidity and higher cost including increased outpatient visits, emergency room visits, treatment with antibiotics, use of home health services, and hospital readmission. ${ }^{6-8}$

CD SSIs can be prevented by adherence to establish perioperative interventions including prophylactic antibiotic administration prior to skin incision. ${ }^{9}$ The effectiveness of perioperative antibiotic administration is contingent on appropriate antibiotic selection, dose, and timing of administration. The American College of Obstetricians and Gynecologists (ACOG) recommends a single dose of perioperative antibiotics within 60 minutes prior to skin incision. ${ }^{10} \beta$-Lactam containing regimens, specifically cephalosporin, are preferred for their gram positive and selective gram negative bacterial coverage. ${ }^{11}$ While the estimated prevalence of penicillin allergy in hospitalized patients is 10 to $20 \%,{ }^{12,13}$ only $12 \%$ of patients who report penicillin allergies are found to be truly allergic. ${ }^{10,12-16} \mathrm{~A}$ received

December 2, 2018 accepted after revision March 8, 2019
DOI https://doi.org/

10.1055/s-0039-1685503. ISSN 2157-6998.
Copyright $\odot 2019$ by Thieme Medical Publishers, Inc., 333 Seventh Avenue, New York, NY 10001, USA. Tel: +1(212) 584-4662.
License terms

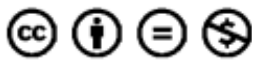


true penicillin allergy is a type I hypersensitivity reaction which is characterized by immediate onset and mediation by IgE (immunoglobulin E) and mast cells and/or basophils (e.g., hives, angioedema, and anaphylaxis). ${ }^{17}$ Of patients with a true penicillin allergy, the cross reactivity to cephalosporin antibiotics is estimated to be 0.17 to $8.5 \%{ }^{12,14,15}$ Given the low rate of cross reactivity, cephalosporins are considered appropriate perioperative prophylaxis in the absence of a type I hypersensitivity to penicillin.

The current recommendation for $\mathrm{CD}$ prophylaxis is a firstgeneration cephalosporin cefazolin. In patients with a type I penicillin allergy, gentamicin and clindamycin are the recommended agents. The purpose of this study was to examine the association between perioperative antibiotic class ( $\beta$-lactam versus non- $\beta$-lactam) and prevalence of $C D$ surgical site infection. In addition, we aimed to assess whether women with penicillin allergies received appropriate perioperative antibiotics when undergoing $\mathrm{CD}$. We hypothesize that women who receive $\beta$-lactam antibiotics, such as cefazolin, prior to CD will have lower odds of SSI compared with those who receive non- $\beta$-lactam antibiotics, such as gentamicin and clindamycin.

\section{Materials and Methods}

The Duke University Health System Institutional Review Board (IRB) approved this retrospective cohort study of women who underwent $C D$ at Duke University Hospital from January 1, 2014 to December 31, 2014.

Women were included in the study if they delivered at $\geq 34$ weeks of gestation and had a documented postpartum visit. Women undergoing $\mathrm{CD}$ received standard preoperative surgical prophylaxis of cefazolin $2 \mathrm{~g}$ intravenously for body mass index (BMI) $<40$ and $3 \mathrm{~g}$ intravenously for $\mathrm{BMI} \geq 40$ with the exception of women with a self-reported penicillin allergy. Penicillin allergy type was defined by medical record allergic event documentation. Women with a penicillin allergy received clindamycin $900 \mathrm{mg}$ intravenously and gentamicin $80 \mathrm{mg}$ intravenously. Subcutaneous closure with absorbable suture was standard practice for women with adipose tissue $\geq 2 \mathrm{~cm}$ in depth. Subcuticular closure with absorbable suture was typically used for skin closure.

Baseline demographic information, medical comorbidities, pregnancy complications, CD indications, and antibiotic use were collected via electronic health record review. Gestational age was determined by best obstetrical estimate using ACOG dating criteria. Pregestational diabetes included patients who had a diagnosis of either type 1 or type 2 diabetes at their obstetric intake visit. Chronic and gestational hypertensions were recorded separately.

The primary outcome of interest was SSI defined by the Centers for Disease Control and Prevention (CDC) as an infection occurring at the surgical site within 30 days of surgery. ${ }^{18}$ All the cases of SSI were confirmed with individual chart review. SSIs include superficial incisional, deep incisional, and organ space infections. For the purposes of our analysis, we categorized postpartum endometritis as a uterine organ space infection diagnosed after hospital discharge and a component of the primary outcome. Endometritis (as opposed to postpartum endometritis) was defined as uterine infection that occurred during the delivery hospitalization and was diagnosed prior to discharge from delivery admission. Due to CDC classification of postpartum as a deep organ space infection, endometritis diagnosed prior to hospital discharge was not included in the primary outcome.

Patients who reported rash or characterized their allergic reaction as "other" were considered low risk. Anaphylaxis, swelling, or an unknown reaction was considered a type I reaction. A woman was considered to receive appropriate antibiotics if she had no or a low-risk reaction to penicillin and was administered a $\beta$-lactam. Similarly, women with a type I reaction to penicillin were considered to receive appropriate antibiotics if they were given a non- $\beta$-lactam.

Summary statistics were calculated for patient and surgery characteristics stratified by antibiotic class. Differences between the two antibiotic groups were tested with Wilcoxon's rank-sum test for continuous variables and either Pearson's Chi-square or Fisher's exact test for categorical variables, as appropriate.

To assess the association between antibiotic class and surgical site infection a logistic regression model was fit controlling for chorioamnionitis in labor, cesarean during labor, endometritis prior to discharge, tobacco use, and delivery BMI. These were clinical factors thought to be related to the treatment assigned and/or surgical site infection. Patients who had missing information for the previously mentioned covariates or penicillin allergy were removed, and a complete case analysis was done due to the low number of missing values. Fisher's exact test was used to assess if the proportion of patients with a type I penicillin allergy that received appropriate antibiotics was different from the proportion in the nonallergic or low-risk group. All statistical analyses were performed in SAS 9.4 (SAS Institute, Cary, NC) at a significance level of 0.05 two-tailed.

\section{Results}

Maternal demographic, clinical, and delivery characteristics are shown in - Table $\mathbf{1}$.

Among the 929 women who delivered by CD, 826 (89\%) received perioperative $\beta$-lactam prophylaxis and 103 (11\%) received prophylaxis with a non- $\beta$-lactam. Of women who received a $\beta$-lactam, $6.7 \%$ developed an SSI compared with $14.6 \%$ of women who received a non- $\beta$-lactam $(p=0.004)$. Patients in the non- $\beta$-lactam group tended to have chronic hypertension and chorioamnionitis more often than the $\beta$ lactam group. The use of Pfannenstiel's skin incision, low transverse uterine incision, subcutaneous sutures, skin closure with suture rather than staples, and negative pressure wound therapy (NPWT) did not differ significantly between the two treatment groups.

In the multivariable logistic regression model, $\beta$-lactam antibiotic prophylaxis was associated with a $57 \%$ reduction in the odds of SSI (odds ratio $[\mathrm{OR}]=0.43 ; 95 \%$ confidence interval $[\mathrm{CI}]=0.22-0.83 ; p=0.01)$ compared with non- $\beta$-lactam prophylaxis (-Table 2 ). Variables significantly associated with 
Table 1 Patient and surgery characteristics by antibiotic class

\begin{tabular}{|c|c|c|c|c|}
\hline & $\beta$-Lactam $(n=826)$ & Non $\beta$-lactam $(n=103)$ & Total $(n=929)$ & $p$-Value \\
\hline Age $(y)$, median $(\mathrm{Q} 1, \mathrm{Q} 3)$ & $31.0(26.0,35.0)$ & $30.0(27.0,35.0)$ & $31.0(26.0,35.0)$ & $0.79^{a}$ \\
\hline Race & & & & $0.85^{\mathrm{b}}$ \\
\hline Non-white & $393(47.6 \%)$ & $50(48.5 \%)$ & $443(47.7 \%)$ & \\
\hline White & $433(52.4 \%)$ & $53(51.5 \%)$ & $486(52.3 \%)$ & \\
\hline Delivery BMI, median (Q1, Q3) & $33.2(28.6,38.9)$ & $34.6(28.0,41.5)$ & $33.4(28.5,39.1)$ & $0.36^{\mathrm{a}}$ \\
\hline Private insurance & $423(51.2 \%)$ & 49 (47.6\%) & $472(50.8 \%)$ & $0.49^{b}$ \\
\hline Gestational age (wk), median (Q1, Q3) & $39.0(37.0,39.0)$ & $39.0(37.0,39.0)$ & $39.0(37.0,39.0)$ & $0.64^{\mathrm{a}}$ \\
\hline Tobacco use & $70(8.5 \%)$ & $9(8.7 \%)$ & $79(8.5 \%)$ & $0.93^{\mathrm{b}}$ \\
\hline Diabetes & $63(7.6 \%)$ & $5(4.9 \%)$ & $68(7.3 \%)$ & $0.31^{\mathrm{b}}$ \\
\hline Missing & 1 & 0 & 1 & \\
\hline Chronic hypertension & $64(7.7 \%)$ & $15(14.6 \%)$ & $79(8.5 \%)$ & $0.02^{\mathrm{b}}$ \\
\hline Gestational hypertension & $141(17.1 \%)$ & $17(16.5 \%)$ & $158(17.0 \%)$ & $0.88^{\mathrm{b}}$ \\
\hline Missing & 1 & 0 & 1 & \\
\hline Cesarean performed in labor & $385(46.6 \%)$ & $54(52.4 \%)$ & $439(47.3 \%)$ & $0.26^{b}$ \\
\hline Chorioamnionitis in labor & $24(2.9 \%)$ & $22(21.4 \%)$ & $46(5.0 \%)$ & $<0.0001^{\mathrm{b}}$ \\
\hline Pfannenstiel incision & $793(96.0 \%)$ & $101(98.1 \%)$ & $894(96.2 \%)$ & $0.42^{c}$ \\
\hline Hysterotomy incision & & & & $0.93^{c}$ \\
\hline Low transverse & $783(94.8 \%)$ & 99 (96.1\%) & 882 (94.9\%) & \\
\hline Classical & $16(1.9 \%)$ & $1(1.0 \%)$ & $17(1.8 \%)$ & \\
\hline Other & $27(3.3 \%)$ & $3(2.9 \%)$ & $30(3.2 \%)$ & \\
\hline Subcutaneous closure & $734(88.9 \%)$ & $88(85.4 \%)$ & $822(88.5 \%)$ & $0.30^{\mathrm{b}}$ \\
\hline Type of closure & & & & $0.57^{c}$ \\
\hline Subcuticular suture & $797(96.5 \%)$ & $101(98.1 \%)$ & $898(96.7 \%)$ & \\
\hline Staples & $29(3.5 \%)$ & $2(1.9 \%)$ & $31(3.3 \%)$ & \\
\hline Negative pressure wound therapy used & $53(6.4 \%)$ & $5(4.9 \%)$ & $58(6.2 \%)$ & $0.54^{b}$ \\
\hline NPWT type & & & & $1.00^{c}$ \\
\hline NPWT not used & 773 & 98 & 871 & \\
\hline Pico & $46(86.8 \%)$ & $5(100.0 \%)$ & $51(87.9 \%)$ & \\
\hline Prevena & $7(13.2 \%)$ & $0(0.0 \%)$ & $7(12.1 \%)$ & \\
\hline Endometritis prior to discharge & $5(0.6 \%)$ & $1(1.0 \%)$ & $6(0.6 \%)$ & $0.51^{c}$ \\
\hline Penicillin allergy & & & & $<0.0001^{\mathrm{b}}$ \\
\hline Nonallergic & 791 (95.8\%) & $21(20.4 \%)$ & $812(87.4 \%)$ & \\
\hline Allergic & $35(4.2 \%)$ & $82(79.6 \%)$ & $117(12.6 \%)$ & \\
\hline Type I penicillin allergy reaction & & & & $<0.0001^{\mathrm{c}}$ \\
\hline No- or low-risk penicillin allergy & 819 (99.2\%) & $74(71.8 \%)$ & $893(96.1 \%)$ & \\
\hline Type I penicillin allergy & $7(0.8 \%)$ & $29(28.2 \%)$ & $36(3.9 \%)$ & \\
\hline
\end{tabular}

Abbreviations: BMI, body mass index; NPWT, negative pressure wound therapy; SSI, surgical site infection; Q1, 1st quartile; Q3, 3rd quartile. aWilcoxon's rank-sum test.

${ }^{b}$ Chi-square test.

'Fisher's exact test.

increased odds of SSI included delivery BMI (OR $=1.03 ; 95 \%$ $\mathrm{CI}=1.01-1.06 ; p=0.01)$, and $\mathrm{CD}$ during labor $(\mathrm{OR}=1.86$; $95 \% \mathrm{CI}=1.10-3.12 ; p=0.02$ ).

The overall prevalence of self-reported allergies was 12.6\%. Among the penicillin-allergic population, $69.2 \%$ of individuals reported a low-risk allergy. In the non- $\beta$-lactam group, $79.6 \%$ reported being allergic to penicillin. Patients with a non-type I penicillin allergy tended to receive appropriate antibiotics more often than those with a type I allergy (91.7 vs. $80.6 \% ; p=0.03$ ). 
Table 2 Association between $\beta$-lactam and surgical site infection controlling for potential confounders

\begin{tabular}{|l|l|l|l|}
\hline Covariate & Unadjusted odds ratio $(95 \% \mathrm{Cl})$ & Adjusted odds ratio $(95 \% \mathrm{Cl})$ & -Value $^{\text {a }}$ \\
\hline Beta-lactam & $0.42(0.23,0.77)$ & $0.43(0.22,0.83)$ & 0.01 \\
\hline Chorioamnionitis & $1.54(0.59,4.02)$ & $0.82(0.28,2.34)$ & 0.70 \\
\hline Cesarean during labor & $1.75(1.06,2.87)$ & $1.86(1.10,3.12)$ & 0.02 \\
\hline Endometritis & $6.29(1.13,34.9)$ & $5.33(0.93,30.8)$ & 0.06 \\
\hline Tobacco use & $1.22(0.54,2.75)$ & $1.06(0.46,2.48)$ & 0.89 \\
\hline Delivery BMI & $1.03(1.01,1.06)$ & $1.03(1.01,1.06)$ & 0.01 \\
\hline
\end{tabular}

Abbreviations: $\mathrm{BMI}$, body mass index; $\mathrm{Cl}$, confidence interval.

${ }^{\mathrm{a}}$ From multivariable logistic regression model.

\section{Discussion}

Our study shows that receiving $\beta$-lactam antibiotics is associated with lower odds of CD SSI compared with non- $\beta$-lactams.

Our findings are consistent with recent gynecologic literature, which suggests that $\beta$-lactam perioperative antibiotics are associated with the lowest risk of SSI compared with alternative regimens. ${ }^{16}$ To date, only one study has investigated the effectiveness of $\beta$-lactam perioperative prophylaxis in obstetric populations. Hopkins et al retrospectively evaluated 22,875 women that delivered via prelabor cesarean and received perioperative antibiotics. The composite primary outcome included wound infection, seroma, hematoma, endometritis, maternal hospital readmission due to a wound complication, and need for wound debridement. In this study, patients who received non- $\beta$-lactam antibiotics were more likely to have a wound complication, including infection, compared with those who received $\beta$-lactam antibiotics ( $14.6 \%$ vs. $6.7 \%$; $p=0.02){ }^{1}{ }^{1}$ Findings from that study are not truly generalizable to present day cesarean surgical practices given the use of staples rather than subcuticular suture for skin closure and antibiotic prophylaxis given after cord clamping rather than preoperatively. ${ }^{1}$

ACOG recommends a single dose of first generation cephalosporin for perioperative prophylaxis at time of cesarean. Cefazolin is a bactericidal antibiotic that provides coverage against common gram positive organisms found in skin flora and many gram negative organisms, such as Escherichia coli. ${ }^{19}$ Gentamicin is also bactericidal and covers gram negative organisms, while clindamycin is bacteriostatic and covers gram positive organisms. As gram positive bacteria are the most common organisms isolated from cesarean wound infection, ${ }^{20}$ the bactericidal nature of cefazolin may help to explain the lower infectious morbidity associated with its use. Another potential contributing factor could be the decreased antibacterial activity of gentamicin and clindamycin $^{21,22}$ that occurs with decreasing tissue $\mathrm{pH}$ and acidic environment that occurs normally at a surgical incision site. ${ }^{23}$

In patients with a type I sensitivity to $\beta$-lactam antibiotics, consideration may be given to penicillin allergy testing to confirm the presence of a true allergy. In women with a confirmed allergy to penicillin, alternative antibiotics must be used, but women receiving the currently recommended antibiotics are at higher risk for postoperative infection than women receiving a first-generation cephalosporin.

The strengths of this study include uniform surgical practice within one institution, perioperative antibiotics prior to skin incision, and documented postpartum followup. These results are generalizable to tertiary care academic medical centers. The limitations of this study include its retrospective design and reliance on self-reported penicillin allergy. In addition, the dose of $80 \mathrm{mg}$ gentamicin is likely suboptimal in a population with a median BMI above 30 and could have contributed to the increased risk of infection seen in the non- $\beta$-lactam group.

Our findings demonstrate the need for educational initiatives regarding appropriate use of antibiotic prophylaxis in patients with a documented $\beta$-lactam allergy. Women with a documented $\beta$-lactam allergy should receive a cephalosporin if the penicillin allergy is not a type I reaction. In addition, allergy testing for women with reported penicillin allergy would greatly increase access to the appropriate perioperative antibiotic for cesarean prophylaxis. In patients that do require non- $\beta$-lactams antibiotics, optimal dosing of both the gentamicin and clindamycin is an important consideration. Further research into alternative non- $\beta$-lactam antibiotic regimens is also warranted.

\section{Paper Presentation}

This paper was presented at Infectious Diseases Society of Obstetrics and Gynecology, Deer Valley, UT, August 10, 2017.

\section{Funding}

The Duke BERD Methods Core's support of this project was made possible (in part) by Grant Number UL1TR002553 from the National Center for Advancing Translational Sciences (NCATS) of the National Institutes of Health (NIH), and NIH Roadmap for Medical Research. Its contents are solely the responsibility of the authors and do not necessarily represent the official views of NCATS or $\mathrm{NIH}$.

\section{Disclosure}

The authors report no conflicts of interest to declare. 


\section{References}

1 Hopkins MK, Dotters-Katz S, Boggess K, Heine RP, Smid M. Perioperative antibiotic choice in labored versus unlabored cesareans and risk of postcesarean infectious morbidity. Am J Perinatol 2018;35(02):127-133

2 Tita AT, Szychowski JM, Boggess K, et al; C/SOAP Trial Consortium. Adjunctive azithromycin prophylaxis for cesarean delivery. $\mathrm{N}$ Engl J Med 2016;375(13):1231-1241

3 Stamilio DM, Scifres CM. Extreme obesity and postcesarean maternal complications. Obstet Gynecol 2014;124(2, Pt. 1):227-232

4 Thigpen BD, Hood WA, Chauhan S, et al. Timing of prophylactic antibiotic administration in the uninfected laboring gravida: a randomized clinical trial. Am J Obstet Gynecol 2005;192(06): 1864-1868, discussion 1868-1871

5 Costantine MM, Rahman M, Ghulmiyah L, et al. Timing of perioperative antibiotics for cesarean delivery: a metaanalysis. Am J Obstet Gynecol 2008;199(03):301.e1-301.e6

6 Johnson A, Young D, Reilly J. Caesarean section surgical site infection surveillance. J Hosp Infect 2006;64(01):30-35

7 Opøien HK, Valbø A, Grinde-Andersen A, Walberg M. Post-cesarean surgical site infections according to $C D C$ standards: rates and risk factors. A prospective cohort study. Acta Obstet Gynecol Scand 2007;86(09):1097-1102

8 Olsen MA, Butler AM, Willers DM, Gross GA, Hamilton BH, Fraser VJ. Attributable costs of surgical site infection and endometritis after low transverse cesarean delivery. Infect Control Hosp Epidemiol 2010;31(03):276-282

9 Sullivan SA, Smith T, Chang E, Hulsey T, Vandorsten JP, Soper D. Administration of cefazolin prior to skin incision is superior to cefazolin at cord clamping in preventing postcesarean infectious morbidity: a randomized, controlled trial. Am J Obstet Gynecol 2007;196(05):455.e1-455.e5

10 American College of Obstetricians and Gynecologists. ACOG Practice Bulletin No. 120: Use of prophylactic antibiotics in labor and delivery. Obstet Gynecol 2011;117(06):1472-1483

11 Faro S, Martens MG, Hammill HA, Riddle G, Tortolero G. Antibiotic prophylaxis: is there a difference? Am J Obstet Gynecol 1990;162 (04):900-907, discussion 907-909
12 Lee CE, Zembower TR, Fotis MA, et al. The incidence of antimicrobial allergies in hospitalized patients: implications regarding prescribing patterns and emerging bacterial resistance. Arch Intern Med 2000;160(18):2819-2822

13 Bourke J, Pavlos R, James I, Phillips E. Improving the effectiveness of penicillin allergy de-labeling. J Allergy Clin Immunol Pract 2015;3(03):365-34.e1

14 Daulat S, Solensky R, Earl HS, Casey W, Gruchalla RS. Safety of cephalosporin administration to patients with histories of penicillin allergy. J Allergy Clin Immunol 2004;113(06): 1220-1222

15 Dash $\mathrm{CH}$. Penicillin allergy and the cephalosporins. J Antimicrob Chemother 1975;1(3, Suppl.):107-118

16 Uppal S, Harris J, Al-Niaimi A, et al. Prophylactic antibiotic choice and risk of surgical site infection after hysterectomy. Obstet Gynecol 2016;127(02):321-329

17 Weiss ME, Adkinson NF. Immediate hypersensitivity reactions to penicillin and related antibiotics. Clin Allergy 1988;18(06): $515-540$

18 Horan TC, Andrus M, Dudeck MA. CDC/NHSN surveillance definition of health care-associated infection and criteria for specific types of infections in the acute care setting. Am J Infect Control 2008;36(05):309-332

19 Faro S. Infectious disease relations to cesarean section. Obstet Gynecol Clin North Am 1988;15(04):685-695

20 Zuarez-Easton S, Zafran N, Garmi G, Salim R. Postcesarean wound infection: prevalence, impact, prevention, and management challenges. Int J Womens Health 2017;9:81-88

21 Lemaire S, Van Bambeke F, Pierard D, Appelbaum PC, Tulkens PM. Activity of fusidic acid against extracellular and intracellular Staphylococcus aureus: influence of $\mathrm{pH}$ and comparison with linezolid and clindamycin. Clin Infect Dis 2011;52(Suppl 7): S493-S503

22 Wagner C, Sauermann R, Joukhadar C. Principles of antibiotic penetration into abscess fluid. Pharmacology 2006;78(01):1-10

23 Woo YC, Park SS, Subieta AR, Brennan TJ. Changes in tissue pH and temperature after incision indicate acidosis may contribute to postoperative pain. Anesthesiology 2004;101(02):468-475 